\title{
SINGLE-QUANTUM NMR SPINNING-SIDE-BAND PATTERNS FOR SPIN-3/2 SYSTEM
}

\author{
A. Lupulescu ${ }^{a, b}$, R. FeChete ${ }^{c}$, D.E. DemCo ${ }^{b, a *}$ AND O. CozAR ${ }^{c}$ \\ aTechnical University, Department of Physics, 3400 Cluj-Napoca, Romania \\ ${ }^{b}$ Max-Planck Institut für Polymerforschung, Postfach 3148, 55021 Mainz, Germany \\ 'Faculty of Physics, University Babes-Bolyai, 3400 Cluj-Napoca, Romania
}

(Received June 24, 1997)

\begin{abstract}
The NMR response of an isolated spin-3/2 system with an axially symmetric quadrupolar interaction under magic-angle sample spinning to an ideal radio-frequency pulse using irreducible tensor operators is evaluated analytically. The excited single-quantum spin coherences are modulated by the sample spinning. The effects of spinning frequency, quadrupole coupling constant and the orientation of quadrupolar interaction relative to rotor axis on the spinning-side-band patterns are simulated. High-resolution single-quantum spinning-side-band pattern detected for ${ }^{23} \mathrm{Na}(I=3 / 2) \mathrm{nu}$ clei in sodium nitrate powder is compared with the simulation based on the theory and the quadrupole coupling is estimated.
\end{abstract}

PACS numbers: $33.25 .+\mathrm{k}, 33.40 .+\mathrm{f}$

\section{Introduction}

High-resolution solid-state NMR spectroscopy is used extensively for studying a great variety of solid materials ranging from ceramics to biopolymers. However, most successful applications have involved spin $I=1 / 2$ nuclei such as ${ }^{13} \mathrm{C}$, ${ }^{15} \mathrm{~N},{ }^{31} \mathrm{P}$, etc. Nevertheless, magic-angle spinning (MAS) NMR spectroscopy of half-integer $(I=3 / 2,5 / 2$, etc.) quadrupolar nuclei in solids is becoming an increasingly popular tool in several physical and materials sciences with ${ }^{27} \mathrm{Al}$ and ${ }^{23} \mathrm{Na}$ representing some of the most important nuclei [1-4] (and references therein). Recent advances in the design of very high-speed $\left(\nu_{\mathrm{R}}<20 \mathrm{kHz}\right)$ and stable $( \pm 5 \mathrm{~Hz})$ spinning devices and probes have proven extremely useful for studies of half-integer quadrupolar nuclei in solids [5-7]. Using these probes, the complete spinning-side-band patterns for the satellite transitions extending over large spectral width were observed for several solids [8-10]. In addition, the computer simulation of the complete spinning-side-band manifolds for the satellite transitions allows quadrupole coupling constant and asymmetry parameters to be determined with high accuracy [10].

\footnotetext{
${ }^{*}$ Corresponding author.
} 
For half-integer quadrupolar nuclei such as ${ }^{23} \mathrm{Na}(I=3 / 2),{ }^{27} \mathrm{Al}(I=5 / 2)$ and ${ }^{17} \mathrm{O}(I=5 / 2)$ improved spectral resolution can be obtained by observing the central $(+1 / 2,-1 / 2)$ transition that is not influenced by the first-order quadrupolar interaction $[11,12]$. However, the spectra are broadened by second-order quadrupolar interaction. This is a fourth rank interaction that cannot be averaged to zero by the MAS technique which is successful in averaging second rank couplings such as dipolar interactions and the chemical shift anisotropy. Several years ago; dynamic-angle spinning (DAS) $[13,14]$ and double-rotation (DOR) $[15-17]$ techniques were developed for obtaining high-resolution isotropic NMR spectra for half-integer quadrupolar nuclei. Unfortunately, both types of experiments are technically demanding and require specially designed NMR probes.

Recently, Frydman and Harwood [18] demonstrated the possibility to obtain isotropic NMR spectra for half-integer quadrupolar nuclei by correlating the evolution of the multiple-quantum (MQ) coherences $(m,-m)$ with that of the single-quantum (SQ) coherences corresponding to the central transition. Structural investigations have been done in the last few years [19-21] using MQ/SQ correlation method which proved to be insensitive to the first- and second-order quadrupolar spectral broadening. Since the above technique involves spin-space manipulation together with sample rotation at the magic angle, it can be implemented easily using an ordinary MAS probe.

The possibility to use high-resolution double-quantum (DQ) spinning-side-band patterns to measure the internuclear distances for protons in rigid solids was proved recently for malonic acid [22]. Moreover, it was shown that the sensitivity of the DQ spinning side-band excited by the recoupling type of pulse sequences to the dipolar couplings can be higher compared to the SQ-spinning-side-band patterns [23]. Of course, it is also important to clarify which strategy is optimal, i.e., to measure the quadrupolar couplings using MQ- or SQ-spinning-side-band patterns or to combine these NMR observables. In order to give a quantitative answer to this problem the first step is to obtain an analytical expression for the SQ-spinning-side-band pattern of an isolated nucleus with quadrupolar interaction. Therefore, the aim of this paper is to evaluate the NMR response of a quadrupolar nucleus with a spin $I=3 / 2$ subjected to MAS, after the excitation by a hard radio-frequency ( $\mathrm{rf}$ ) pulse. The simulation based on the analytical expression for SQ spinning-side-band patterns are compared with the experimental data recorded for ${ }^{23} \mathrm{Na}$ in $\mathrm{NaNO}_{3}$ powder.

\section{The Hamiltonian}

In the strong external magnetic field the Hamiltonian for a spin- $I$ quadrupolar nucleus subjected to magic-angle sample spinning may be approximated by the secular first-order average Hamiltonian over the Larmor period

$$
\begin{aligned}
H_{Q}(t) & =\sum_{m=-2}^{2} \hbar \omega_{Q}^{(m)} \exp \left(\mathrm{i} m \omega_{\mathrm{R}} t\right)\left[3 I_{z}^{2}-I(I+1)\right] \\
& =\sum_{m=-2}^{2} \hbar \sqrt{6} \omega_{Q}^{(m)} \exp \left(\mathrm{i} m \omega_{\mathrm{R}} t\right) T_{2,0}
\end{aligned}
$$


In the above equation $\omega_{R} / 2 \pi$ is the MAS frequency and $T_{2,0}$ is the irreducible tensor operator of rank two and order zero. $T_{2,0}$ is given by

$$
T_{2,0}=\frac{1}{\sqrt{6}}\left[3 I_{z}^{2}-I(I+1)\right] \text {. }
$$

The spatial dependence of the quadrupolar Hamiltonian is written in terms of its Fourier coefficients as

$$
\begin{aligned}
\omega_{Q}^{(m)} & =\omega_{Q}^{\max }\left\{D_{0,-m}^{2}\left(\Omega_{\mathrm{PR}}\right)-\frac{\eta}{\sqrt{6}}\left[D_{-2,-m}^{2}\left(\Omega_{\mathrm{PR}}\right)+D_{-2, m}^{2}\left(\Omega_{\mathrm{PR}}\right)\right]\right\} \\
& \times d_{-m, 0}^{2}\left(\beta_{\mathrm{RM}}\right) .
\end{aligned}
$$

The maximum quadrupole splitting is

$$
\omega_{Q}^{\max }=\frac{2 \pi C_{Q}}{4 I(2 I-1)},
$$

where $C_{Q}=e^{2} q Q / h$, is the quadrupole coupling constant and $\eta$ is the electric field gradient asymmetry parameter $(0 \leq \eta \leq 1)$. $D_{k, l}^{2}$ is the Wigner matrix with $\Omega_{\mathrm{PR}}=\{\alpha, \beta, \gamma\}$ denoting Euler angles relating the rotor-fixed frame $\mathrm{R}$ to the principal-axis frame $(\mathrm{P})$ of the quadrupole interaction, whereas $d_{k, l}^{2}$ is the reduced Wigner matrix element having the argument $\beta_{\mathrm{RL}}=\beta_{\mathrm{M}}=\tan ^{-1}(1 / \sqrt{2})$ for MAS, relating $R$ to the laboratory frame $L$.

In the following we shall consider the simplified case of axially symmetric quadrupole interaction, i.e., $\eta=0$. From Eq. (3) we have

$$
\omega_{Q}^{(m)}=\omega_{Q}^{\max } D_{0,-m}^{2}\left(\Omega_{\mathrm{PR}}\right) d_{-m, 0}^{2}\left(\beta_{\mathrm{RL}}\right) \text {. }
$$

Taking into account that at the magic angle $\omega_{Q}^{(0)}=0$, we can write for the rotor modulated quadrupolar Hamiltonian

$$
\begin{aligned}
& H_{Q}(t)=\hbar \sqrt{6}\left[\omega_{Q}^{(1)} \exp \left(\mathrm{i} \omega_{\mathrm{R}} t\right)+\omega_{Q}^{(-1)} \exp \left(-\mathrm{i} \omega_{\mathrm{R}} t\right)+\omega_{Q}^{(2)} \exp \left(\mathrm{i} 2 \omega_{\mathrm{R}} t\right)\right. \\
& \left.\quad+\omega_{Q}^{(-2)} \exp -\left(\mathrm{i} 2 \omega_{\mathrm{R}} t\right)\right] .
\end{aligned}
$$

Using Eqs. (3) and (4) we can finally write

$$
\begin{gathered}
H_{Q}(t)=\hbar \omega_{Q}^{\max }\left[-\sqrt{3} \sin 2 \beta \cos \left(\omega_{\mathrm{R}} t+\gamma\right)\right. \\
\left.+\sqrt{\frac{3}{2}} \sin ^{2} \beta \cos \left(2 \omega_{\mathrm{R}} t+2 \gamma\right)\right] T_{2,0},
\end{gathered}
$$

or written in a short-hand notation

$$
H_{Q}(t)=\hbar \omega_{Q}(t) T_{2,0} \text {. }
$$

In the argument of the free evolution period propagator acting during the period of time $(0, t)$, the integrated quadrupolar Hamiltonian is

$$
H_{Q}(t, 0)=\int_{0}^{t} H_{Q}(t) \mathrm{d} t=\frac{\hbar \omega_{Q}(t ; 0)}{\sqrt{6}} T_{2,0},
$$

where 


$$
\begin{gathered}
\omega_{Q}(t ; 0)=\sqrt{6} \frac{\omega_{Q}^{\max }}{\omega_{\mathrm{R}}}\left\{-\sqrt{3} \sin 2 \beta\left[\sin \left(\omega_{\mathrm{R}} \mathrm{t}+\gamma\right)-\sin \gamma\right]\right. \\
\left.+\sqrt{\frac{3}{2}} \sin ^{2} \beta\left[\sin \left(2 \omega_{\mathrm{R}} t+2 \gamma\right)-\sin (2 \gamma)\right]\right\} .
\end{gathered}
$$

It is easy to generalize the above formulae for the case of quadrupole interaction with $\eta \neq 0$.

\section{Single-quantum spinning-side-band pattern}

We shall consider in the following a $I=3 / 2$ quadrupolar nucleus in a rigid lattice for which the chemical shielding interaction as well as internuclear dipolar couplings can be neglected.

The relevant equilibrium spin density operator is $\rho_{\mathrm{eq}} \approx C I_{z}$, where $C$ is the Curie factor. In the limit of hard radio-frequency $\pi / 2$ pulse the density operator at the beginning of free induction decay (FID) is given by

$$
\rho(0)=\exp \left(\mathrm{i} \frac{\pi}{2} \widehat{I}_{x}\right) I_{z}=\mathrm{i} T_{1,1}(s)
$$

where the symmetric irreducible tensor operator is defined as $T_{1,1}(s)=$ $\frac{1}{\sqrt{2}}\left(T_{1,1}+T_{1,-1}\right)$ with $T_{1, \pm 1}=\mp\left(\frac{1}{2}\right)^{1 / 2} I_{ \pm}$and $I_{ \pm}=I_{x} \pm \mathrm{i} I_{y}[24,25]$. The Liouville propagator is $\exp (\mathrm{i} \widehat{O}) A=\exp (\mathrm{i} O) A \exp (-\mathrm{i} O)$.

The propagator which describes the spin system free evolution in the presence of MAS can be written as

$$
E(t ; 0)=T \exp \left(-\frac{\mathrm{i}}{\mathrm{h}} \int_{0}^{t} H_{Q}(t) \mathrm{d} t\right) .
$$

From Eq. (9) the above equation can be rewritten as

$$
E(t ; 0)=\exp \left(-i \frac{\omega_{Q}(t ; 0)}{\sqrt{6}} T_{2,0}\right) \text {. }
$$

The density operator, which describes the FID, is given by

$$
\rho(t)=\exp \left(-\mathrm{i} \frac{\omega_{Q}(t ; 0)}{\sqrt{6}} \widehat{T}_{2,0}\right) \rho(0)=\mathrm{i} C \exp \left(-\mathrm{i} \frac{\omega_{Q}(t ; 0)}{\sqrt{6}} \widehat{T}_{2,0}\right) T_{1,1}(s) \text {. }
$$

The above density operator for arbitrary moment of time can be evaluated using the evolution of the irreducible tensor operators under a quadrupole interaction for spin $I=3 / 2$ [25]. We finally get

$$
\begin{aligned}
\rho(t) & =C\left\{\mathrm{i} T_{1,1}(s)\left[\frac{3}{5} \cos \left(\omega_{Q}(t ; 0)\right)+\frac{2}{5}\right]+T_{2,1}(a) \frac{1}{\sqrt{2}} \sin \left(\omega_{Q}(t ; 0)\right)\right. \\
& \left.+T_{3,1}(s) \frac{2}{\sqrt{15}}\left[\cos \left(\omega_{Q}(t ; 0)\right)-1\right]\right\} .
\end{aligned}
$$

It is evident from this relationship that after the action of a radio-frequency pulse the spin system develops only SQ coherences, i.e., the density operator contains only terms,proportional with $I_{ \pm}$raising and lowering operators. 
The normalized NMR signals detected in the rotating frame can be evaluated from the relationships

and

$$
S_{x}(t)=\frac{\operatorname{Tr}\left\{I_{x} \rho(t)\right\}}{\operatorname{Tr}\left\{I_{z} \rho_{\mathrm{eq}}\right\}}
$$

$$
S_{y}(t)=\frac{\operatorname{Tr}\left\{I_{y} \rho(t)\right\}}{\operatorname{Tr}\left\{I_{z} \rho_{\mathrm{eq}}\right\}} .
$$

These orthogonal signals can be evaluated using Eq. (15) and the traces of the irreducible tensor operator products given in Ref. [25]. We can write

$$
S_{x}(t)=0,
$$

and

$$
S_{y}(t)=\frac{1}{5}\left[3 \cos \left(\omega_{Q}(t ; 0)\right)+2\right] .
$$

The Fourier transform of the FID, Eq. (17b), will lead to the spinning-side-band pattern. The relative intensities of the various spinning-side-bands can be analytically evaluated using a Fourier-Bessel series.

The FID derived above is valid for a single crystal. For a powdery solid the FID is given by

$$
S_{y}(t)=\frac{1}{5}\left[3\left\langle\cos \left(\omega_{Q}(t ; 0)\right)\right\rangle+2\right],
$$

where the powder average is

$$
\begin{aligned}
& \left\langle\cos \left(\omega_{Q}(t ; 0)\right)\right\rangle=\frac{1}{8 \pi^{2}} \int_{0}^{2 \pi} \mathrm{d} \alpha \int_{0}^{\pi} \sin \beta \mathrm{d} \beta \int_{0}^{2 \pi} \mathrm{d} \gamma \cos \left(\omega_{Q}(t ; 0)\right) \\
& =\frac{1}{4 \pi} \int \sin \beta \mathrm{d} \beta \int_{0}^{2 \pi} \mathrm{d} \gamma \cos \left(\omega_{Q}(t ; 0)\right) .
\end{aligned}
$$

\section{Results and discussion}

The effect of the quadrupole couplings, the orientation of the quadrupole axially symmetric tensor relative to the rotor reference frame and rotor frequency on the SQ spinning-side-band patterns can be analyzed using Eq. (17b) by numerical simulations.

The spinning-side-band patterns for different values of the quadrupole coupling constant $C_{Q}$ in the range $300 \mathrm{kHz}-1 \mathrm{MHz}$ are presented in Fig. 1. As the quadrupole coupling constant is increased the relative intensities of the spinning side-bands relative to the center band are decreasing but the features of triplet static NMR spectrum are present. For the strongest value of the $C_{Q}$ the spinning-side-band pattern is extended to a broader spectral range compared to the weaker coupling. If this spectrum could be recorded the quadrupole coupling constant can be measured with better accuracy as a result of more rotor lines in the spinning-side-band pattern.

The dependence of the patterns versus the $\beta$ angle for $\gamma=0^{\circ}$ and a quadrupole coupling constant of $C_{Q}=337 \mathrm{kHz}$ corresponding to ${ }^{23} \mathrm{Na}$ nuclei in $\mathrm{NaNO}_{3}$ are presented in Fig. 2. The structure with high intensity spinning-side-bands 

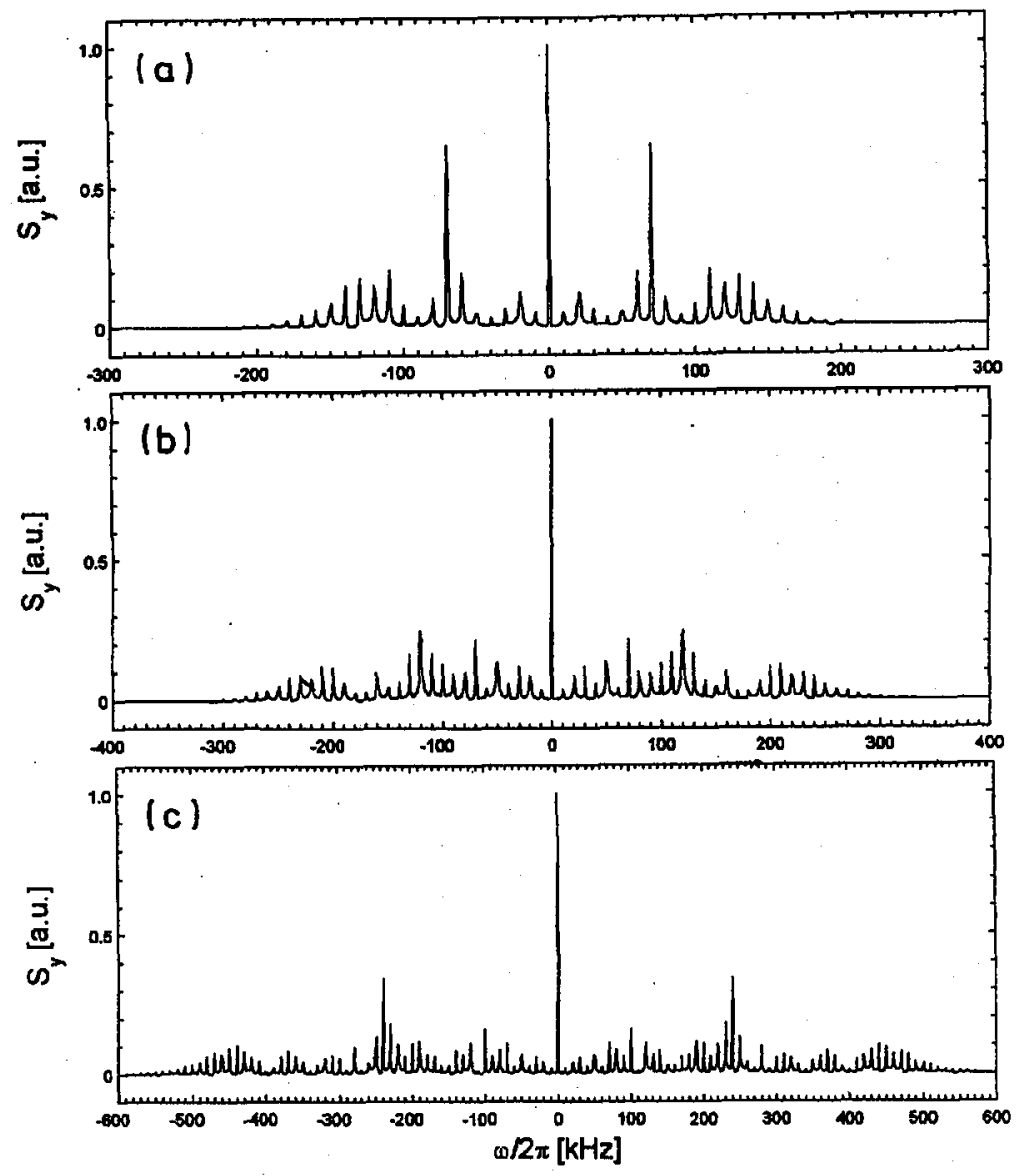

Fig. 1. Spinning-side-band pattern simulations from Eq. (17b), for $\beta=45^{\circ}, \gamma=0^{\circ}$, $\omega_{\mathrm{R}} / 2 \pi=10 \mathrm{kHz}$ and quadrupole coupling constant $C_{Q}=$ (a) $300 \mathrm{kHz}$, (b) $500 \mathrm{kHz}$ and (c) $1 \mathrm{MHz}$, respectively.

is evident for the polar angle $\beta=30^{\circ}$ as compared with the other two angles. Nevertheless, the spinning-side-band pattern will vanish for $\beta=0^{\circ}$ as it is evident from Eq. (10). As the rotor polar angle is increased the intensities of the spinning-side-bands will decrease but the structure will become richer.

The dependence of spinning-side-band patterns versus the rotor frequencies is presented in Fig. 3. For the highest rotor frequency considered of $\omega_{\mathrm{R}}=25 \mathrm{kHz}$

- the relative intensities of the spinning-side-bands to the center band are higher compared with the cases of lower rotor frequency. The advantage of high sensitivity and of resolution offered by fast MAS is supplemented by the higher sensitivity of the spinning-side-bands patterns to the quadrupole couplings.

The SQ spinning-side-band pattern for ${ }^{23} \mathrm{Na}$ in powder $\mathrm{NaNO}_{3}$ (obtained commercially and used without additional purification) is presented in Fig. 4a. 

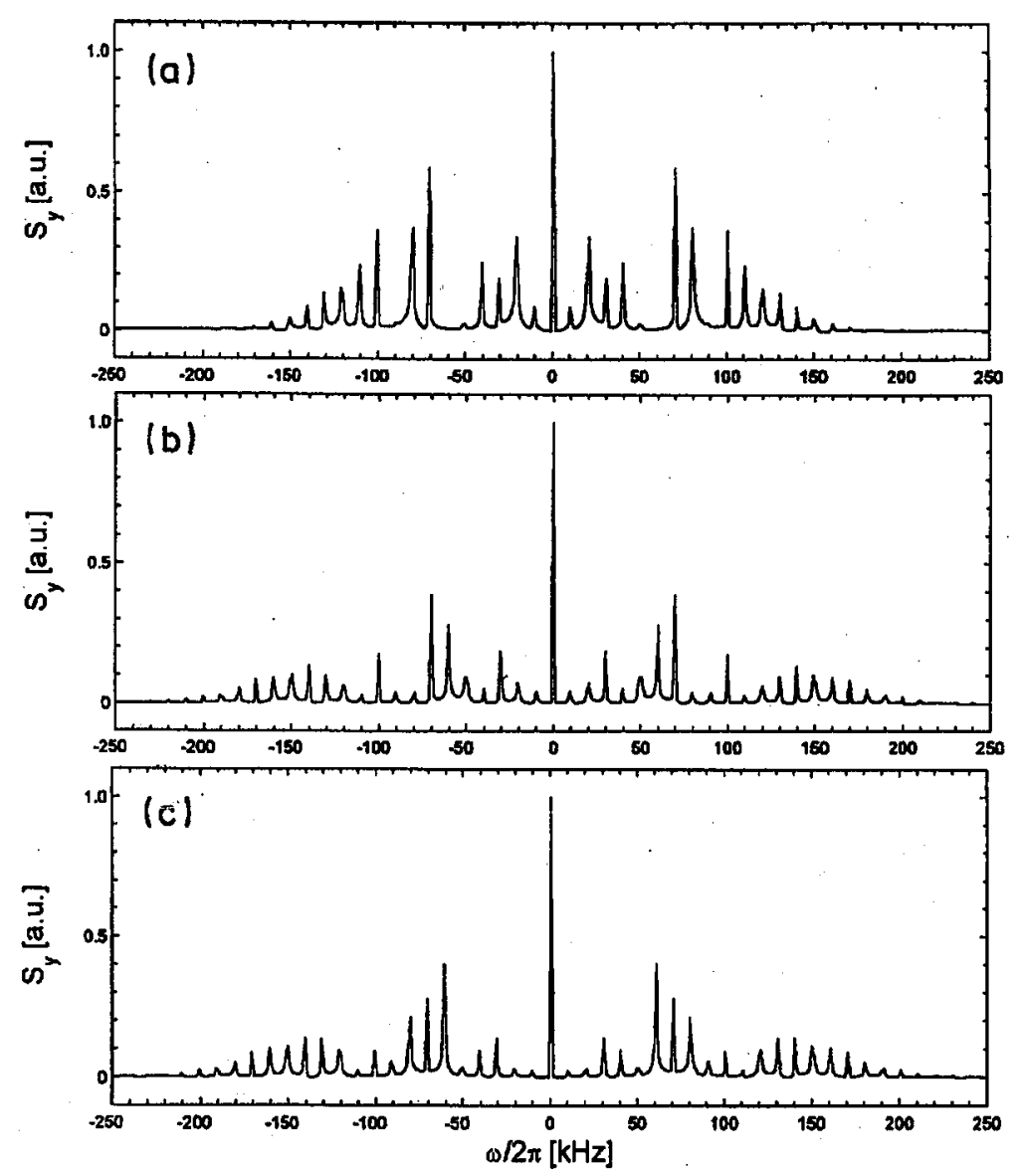

Fig. 2. Spinning-side-band pattern simulations from Eq. (17b), for $C_{Q}=337 \mathrm{kHz}$, $\gamma=0^{\circ}, \omega_{\mathrm{R}} / 2 \pi=10 \mathrm{kHz}$ and for rotor polar angle $\beta=30^{\circ}$ (a), $54.7^{\circ}$ (b) and $60^{\circ}$ (c).

The measurements were performed on a Bruker ASX 500 NMR spectrometer operating at a proton frequency of $500.13 \mathrm{MHz}$. The commercial double-tuned $4 \mathrm{~mm}$ MAS probe has been used with a $90^{\circ}$ pulse length of $1.5 \mu \mathrm{s}$ at the ${ }^{23} \mathrm{Na}$ resonance frequency. The simulation of the spinning-side-band pattern was performed by averaging the FID over all the crystallite orientations by multiplication with a Gaussian decay and finally by Fourier transformation. The simulation presented in Fig. 4b used Eqs. (18) and (19) with quadrupole coupling constant of $C_{Q}=337 \mathrm{kHz}$ and asymmetry parameter $\eta=0.00$ and a Gaussian line width of $120 \mathrm{~Hz}$ [26]. The simulation reproduces well the experimental spectrum but the intensities of the spinning-side-bands are a little higher for the experimental spectrum. We believe that this effect is related mainly to the finite radio-frequency excitation pulse. 


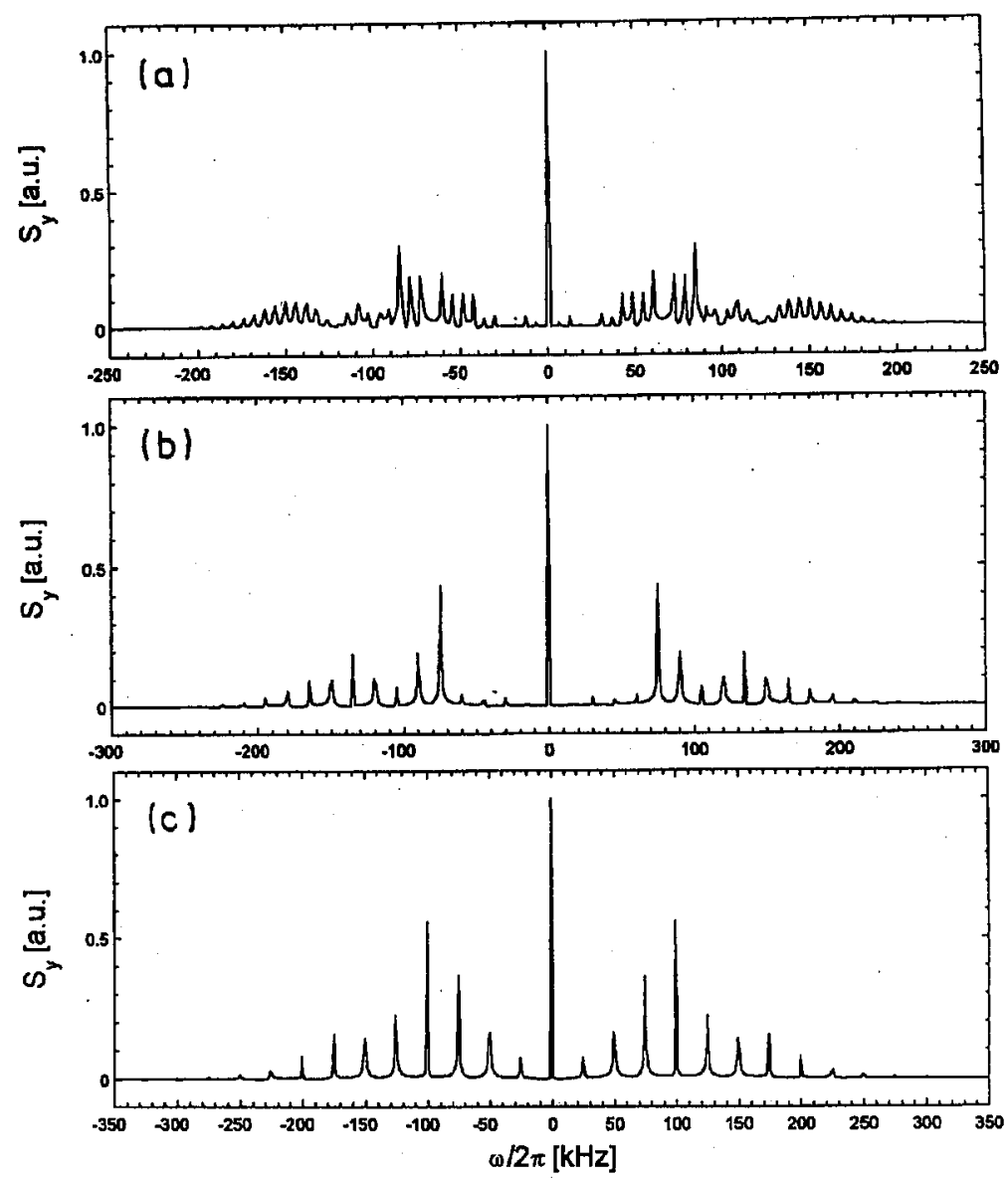

Fig. 3. Spinning-side-band pattern simulations from Eq. (17b), for $C_{Q}=337 \mathrm{kHz}$, $\beta=45^{\circ}$ and $\gamma=0^{\circ}$ and quadrupole coupling constant $\omega_{\mathrm{R}}=6 \mathrm{kHz}(\mathrm{a}), 15 \mathrm{kHz}$ (b) and $25 \mathrm{kHz}(\mathrm{c})$.

\section{Conclusions and outlook}

The spinning-side-band patterns for a spin $I=3 / 2$ with an axially symmetric quadrupolar coupling in the secular approximation were evaluated using the elegant formalism of the density operator together with the algebra of rotation of the irreducible tensor operators. This formalism can be extended to the case of the anisotropic quadrupole interaction and to the radio-frequency excitation pulses having finite duration.

The effect of the rotor frequency, the strength of quadrupole interaction and the orientation of quadrupole tensor on the spinning-side-band patterns was investigated by simulations for the particular case of ${ }^{23} \mathrm{Na}$ nuclei in $\mathrm{NaNO}_{3}$. This will open the possibility to discuss the changes of the patterns for the sample 

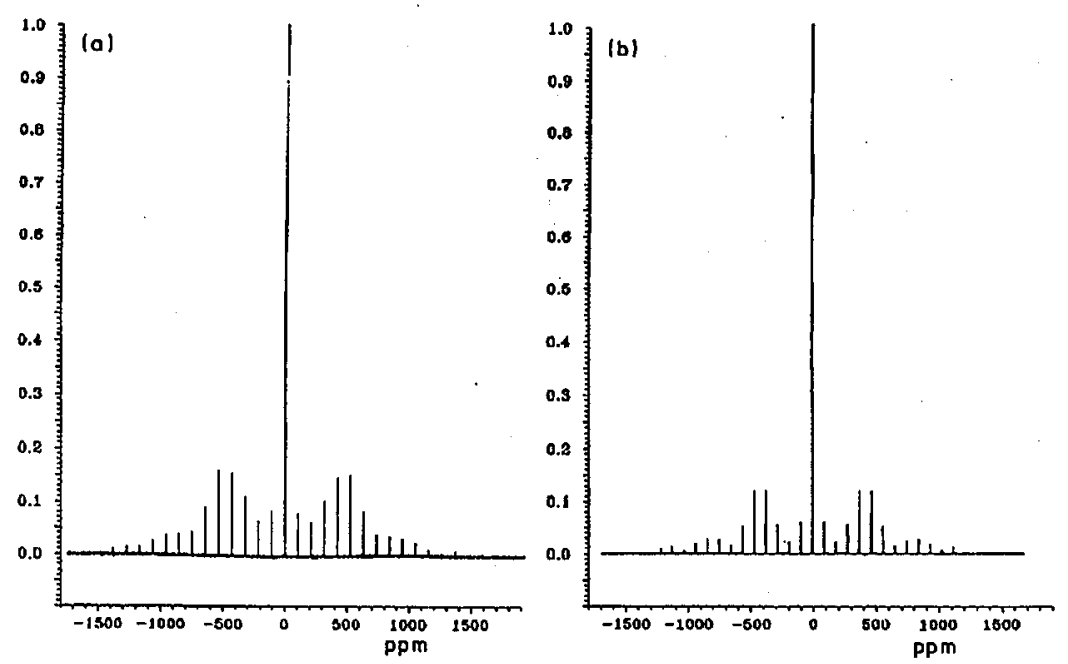

Fig. 4. (a) ${ }^{23} \mathrm{Na}$ spinning-side-band pattern for powder ${ }^{23} \mathrm{NaNO}_{3}$ recorded after a. $90^{\circ}$ radio-frequency pulse of $1.5 \mu \mathrm{s}$ at a proton resonance frequency of $500.13 \mathrm{MHz}$. (b) Simulated spinning-side-band pattern for ${ }^{23} \mathrm{Na}$ with quadrupole coupling constant of $C_{Q}=337 \mathrm{kHz}$ and asymmetry parameter $\eta=0.00$ and a Gaussian line-width of $120 \mathrm{~Hz}[10,26]$.

rotation at an angle different from the magic angle.

The presented results can be extended directly to the case of isolated $\mathrm{CH}_{3}$ -group undergoing rapid classical rotation. The rotating $\mathrm{CH}_{3}$ spin system in the high magnetic field can be regarded as a sum of three quasiparticles: one quadrupolar-like quasiparticle of spin $3 / 2$ and two quasiparticles of spin $1 / 2[27,28]$. The motional averaged dipolar couplings can be evaluated directly from the spinning-side-band patterns using a relationship similar with that derived for the case of a quadrupole nucleus.

Triple-quantum (TQ) spinning-side-band patterns excited by a three-pulse sequence can be evaluated in the same manner as that presented above. The sensitivity of the TQ and the SQ spinning-side-band patterns to a distribution of quadrupole couplings can be also investigated using the results presented in this paper. Work along this line is currently underway.

\section{Acknowledgments}

The authors acknowledge financial support from Volkswagen-Stiftung. The authors thank Prof. Dr. Hans W. Spiess, Dr. Siegfried Hafner and Ulli Friedrich for helpful comments. 


\section{References}

[1] D. Müller, W. Gessner, H.-J. Behrens, G. Scheller, Chem. Phys. Lett. 79, 59 (1981).

[2] M.D. Meadows, K.A. Smith, R.A. Kinsey, T.M. Rothgeb, R.P. Skarjune, E. Oldfield, Proc. Natl. Acad. Sci. USA 79, 1351 (1982).

[3] A. Samorson, E. Lippmaa, Phys. Rev. B 28, 6567 (1983).

[4] D. Freude, J. Haase, NMR Basic Principles and Progress 29, 3 (1993).

[5] P. Daugaard, V. Langer, H.J. Jakobsen, J. Magn. Reson. 70, 472 (1986).

[6] P. Daugaard, V. Langer, H.J. Jakobsen, J. Magn. Reson. 76, 161 (1988).

[7] L.B. Alemany, H.K.C. Timken, D. Johnson, J. Magn. Reson. 80, 427 (1988).

[8] A. Samoson, Chem. Phys. Lett. 119, 29 (1985).

[9] E. Lippmaa, A. Samoson, M. Mägi, J. Am. Chem. Phys. 108, 1730 (1986).

[10] H.J. Jakobsen, J. Skibsted, H. Bildsoe, N.Chr. Nielsen, J. Magn. Reson. 85, 173 (1989).

[11] E. Kundla, A. Samoson, E. Lippmaa, Chem. Phys. Lett. 83, 229 (1981).

[12] S. Ganapathy, S. Schramm, E. Oldfield, J. Chem. Phys. 77, 4360 (1982).

[13] A. Llor, J. Virlet, Chem. Phys. Lett. 151, 470 (1988).

[14] K.T. Meuller, B.Q. Sun, G.C. Chingas, J.W. Zwanziger, T. Terao, A. Pines, J. Magn. Reson. 86, 470 (1990).

[15] A. Samoson, E. Lippmaa, A. Pines, Mol. Phys. 65, 1013 (1988).

[16] B.F. Chmelka, K.T. Mueller, A. Pines, J. Stebbins, Y. Yu, J.W. Zwanziger, Nature 339, 42 (1989).

[17] Y. Yu, B.Q. Sun, A. Pines, A. Samoson, E. Lippmaa, J. Magn. Reson. 89, 296 (1990).

[18] L. Frydman, J.S. Harwood, J. Am. Chem. Phys. 67, 1752 (1995).

[19] C. Fernandez, J.P. Amoreux, Chem. Phys. Lett. 242, 449 (1995).

[20] S.P. Brown, S.J. Heyes, S. Wimperis, J. Magn. Reson. A 119, 280 (1996).

[21] G. Wu, D. Rovnyank, B. Sun, R.G. Griffin, Chem. Phys. Lett. 249, 210 (1995).

[22] J. Gottwald, D.E. Demco, R. Graf, H.W. Spiess, Chem. Phys. Lett. 243, 314 (1995).

[23] R. Graf, D.E. Demco, J. Gottwald, S. Hafner, H.W. Spiess, J. Chem. Phys. 106, 885 (1997).

[24] G.J. Bowden, W.D. Hutchinson, J. Magn. Reson. 67, 403 (1986).

[25] G.J. Bowden, W.D. Hutchinson, J. Magn. Reson. 67, 415 (1986).

[26] J. Skibstead, N.C. Nielsen, H. Bildsoe, H.J. Jacobsen, J. Magn. Reson. 95, 88 (1991).

[27] P.S. Allen, W. Harding, P. Mansfield, J. Phys. C, Solid State Phys. 5, 189 (1972).

[28] Yu.N. Moskvich, N.A. Sergeev, G.I. Dotsenko, Phys. Status Solidi A 30, 409 (1975). 